\title{
Proceeding
}

Supplementary Issue: Summer Conferences of Sports Science. Costa Blanca Sports Science Events, 20-21 September 2019.

Alicante, Spain.

\section{A study of clumsiness and sport}

\section{FRANCESCO PERROTTA ${ }^{1}$, ROBERTA ROSA ${ }^{2}$, GIUSEPPE MADONNA ${ }^{3}$}

${ }^{1}$ M.I.U.R. Campania, Italy

2IUL Telematic University, Italy

${ }^{3}$ Parthenope University, Naples, Italy

\begin{abstract}
Every sport that is known, prevents a series of skills, real or acquired over time, which aim at the explosiveness of the gesture, looking for the maximum possible performance. The aim of this research work is to analyze a very important element, but very underrated: the clumsiness, which is able to change the result of a sport competition and which can be defined, in every sport, the enemy of every athlete. Keywords: Clumsiness; Athletic; Performance.
\end{abstract}

\section{Cite this article as:}

Perrotta, F., Rosa, R., \& Madonna, G. (2019). A study of clumsiness and sport. Journal of Human Sport and Exercise, 14(5proc), S1977-S1985. doi:https://doi.org/10.14198//hse.2019.14.Proc5.18

Corresponding author. M.I.U.R. Campania, Italy.

E-mail: giuseppe.madonna1984@gmail.com

Supplementary Issue: Summer Conferences of Sports Science. Costa Blanca Sports Science Events, 20-21 September 2019. Alicante, Spain.

JOURNAL OF HUMAN SPORT \& EXERCISE ISSN 1988-5202

(c) Faculty of Education. University of Alicante

doi:10.14198/jhse.2019.14.Proc5.18 


\section{PURPOSE OF STUDY}

Looking for the reasons for athletic clumsiness starting from the base of all this, that is, where does physical clumsiness come from? And why so much developed in the modern era? We will find the answers to solve this problem.

\section{RECENT FINDINGS}

One aspect of daily life that is strongly affected by this situation is precisely the education to the movement to a healthy and active life, that is to say all this affects the child's movement and body, as it is, both in the school context and in those outside the school, motor-sports culture, in addition to being insufficient and not adequately promoted, is still confined to a practical-training role, where what counts is the result.

Through the various studies included in this systematic research we wanted to highlight how education to the movement, if implemented since pre-school age, can positively influence all the facets of the personality of each individual, and how body and movement are useful and necessary for structuring cognitive, affective, social and moral skills.

Keywords: Evolutive Age, Pedagogy, Physical Activity, Development of Personality, Gaming and Socialization, Motor-Motor Development, Early Education physical activity in developmental age is an essential element. Today we find ourselves at the centre of a vortex caused by a crisis, now global, which affects not only the economic and political aspect of nations but is also affecting the ethical and cultural development of the population.

The great importance of physical activity in today's society is highlighted by the presence of infrastructures, environments, and events, which are totally focused on the promotion of active lifestyles and motor development. Despite this, for many years and still today, despite fighting inactivity and knowing what damage it brings to the population, unfortunately it is too early to talk about an active world society, or rather, there are few States that give the right importance and regularly encourage motoring education in young people, implementing it with school and extra-school activities as a fundamental discipline for the development of the child.

Unfortunately, Italy is not one of these countries. The most fitting demonstration is provided by the information sheet no. 311 on obesity and overweight (WHO, 2013), finding 42 million children in conditions of obesity and overweight, of which 35 million belong to developing countries; this means that in many states the "double impact" is fought, if on the one hand in the extreme periphery one dies of poverty and hunger, in the most industrialized areas the opposite happens; moreover, one adult in three in the world is not sufficiently active. In $56 \%$ of the member states of the World Health Organization, policies are in place to combat physical inactivity; the latter have agreed to reduce physical inactivity by $10 \%$ by 2025 (Demofanti, Ministry of Health, 2014).

The Italian situation can be an obvious example of how physical education is devalued and we cannot pour all the blame only on the institutions, we must be educators, as well as families, to question and reflect on how we propose but above all how we report to our children about motor activity; from the data provided by the Save The Children (2012) research, $73 \%$ of parents declare that their children spend very little time outdoors, due to lack of space and safety, plus one child in four passes at least three hours a day in front of 
the TV, the videogames or the computer; a good $57 \%$ of parents of obese or overweight and inactive children do not believe it is necessary for their children to incentivize and increase their level of physical activity.

Nowadays moving seems to have become a serious problem, and to think that it should represent the most natural act of man, the moment has arrived to give a "shock" to this society that does not want to invest in the movement. To develop the research, to use the movement as an educational means to improve the motor and cognitive development of the child with benefits not only physical but also in the most intimate processes of the person. Physical exercise promoted since pre-school age has a positive influence on our organs and apparatuses with improvements and a lower incidence of pathologies in the various systems: cardio-vascular, endocrinological, respiratory, osteo-articular, metabolic, but to stop this would be limiting, since it also affects the personality acting on the superior cortical structures modulating our socio-relational, emotional, emotional, intellectual behaviours (WHO, 2007, MIUR, 2012). In evolutive age, corporeity acquires an unbearable meaning, that set of concrete and abstract elements begins to come to life, sketches a communication, in fact it is with this very special medium that the child expresses his emotions and his 'agnost, undertakes relationships with others, acquires knowledge and experience and uses it as a shield, a sort of shell to be able to detach from a reality that sometimes seems to scare him.

As we see, nowadays physical activity has drastically reduced, reaching the historical lows, where obesity and the incorrect lifestyle are to be found in at least $3 / 5$ people. One of the biggest problems is the absence of certain public facilities that allow free play and entertainment through motor activity, which in children has proved to be totally necessary, since the various neuromotor processes require stimulation to coordinate gestures and the skeletal musculature to make movements, even the simplest ones.

In fact, the motor neurons of skeletal muscles and gill muscles are monosynaptic. The effector pathway instead of smooth muscle tissue is un-synaptic (it involves two neurons: the "visceral general motor neurone" located in the CNS which engages on a ganglionic neuron which innervates the muscle.).

All motoneurons from the central nervous system must first contract synapses in the spinal cord, then go to the target muscle.

Each somatic motor neuron Aa which exits the marrow to go to the relative muscle emits a recurrent axon returning to the medulla with synapse with an inhibitory interneuron of the lamina VII (Renshaw neuron, which discharges on the same motoneuron and on synergic motoneurons of adjacent neuromeres). This retrograde inhibition system eliminates the possibility of overstimulation of a muscle, which is commonly called tetanic stimulation, in some cases physiological (example: maximal isometric contraction) (Figure 1 and Figure 2).

Coordination is the first maturing process of the motor action that consists of a progressive tonic-dynamic regulation between the agonist and antagonist muscles of the different body segments, interested in that given movement, to achieve a correct spatial organization of the intentional act. The child, during the learning phase of that given motor scheme, repeats it numerous times even in the following days until obtaining the result considered optimal by him in relation to the desire. In the first experiments the act is insecure, badly organized in a temporal-spatial sense, now with slow moments, sometimes brusque, but the frequent repetition of the tests improves the result and allows the progressive improvement of the motor scheme in which the child has exercised. 


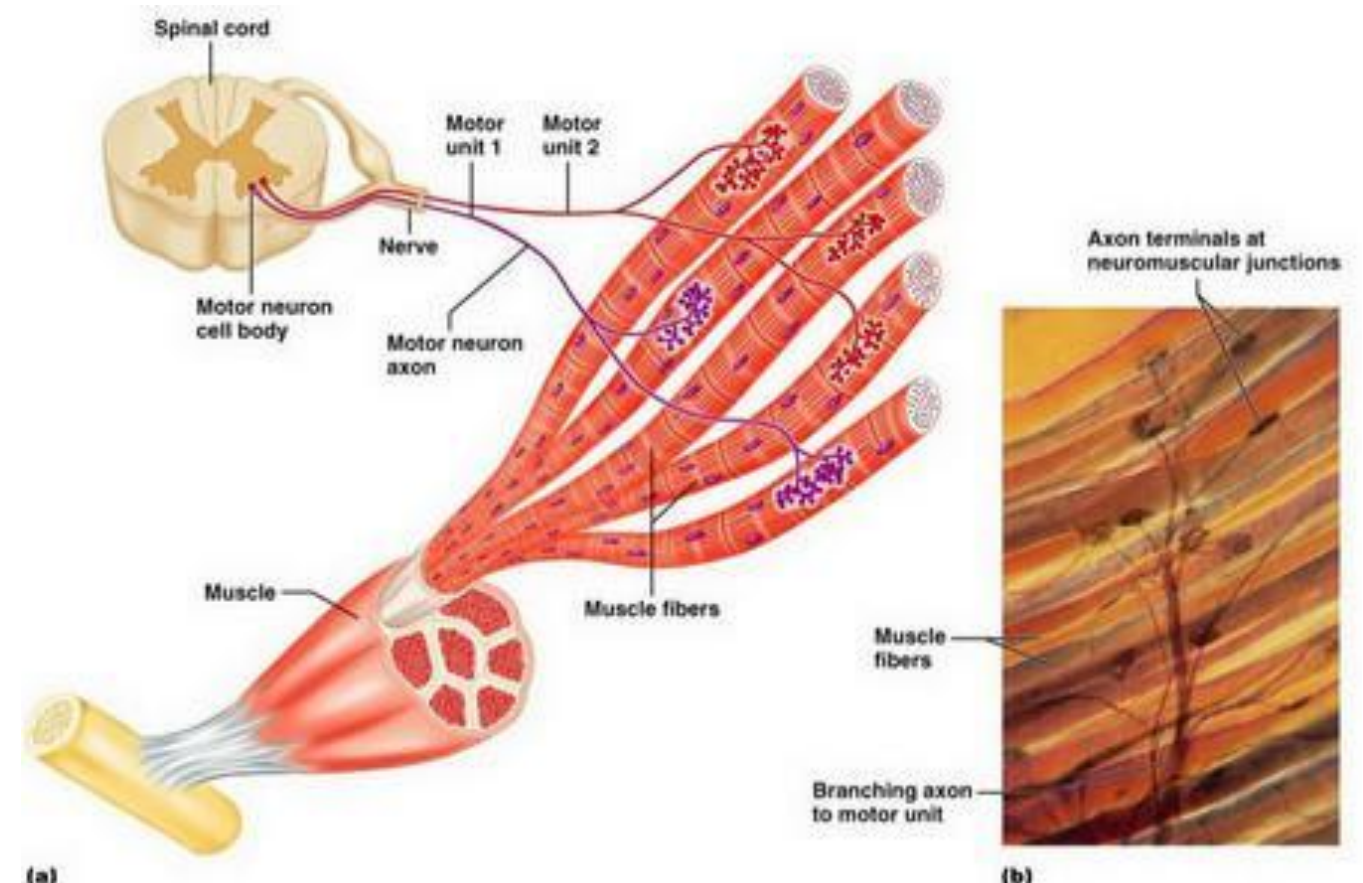

(a)

(b)

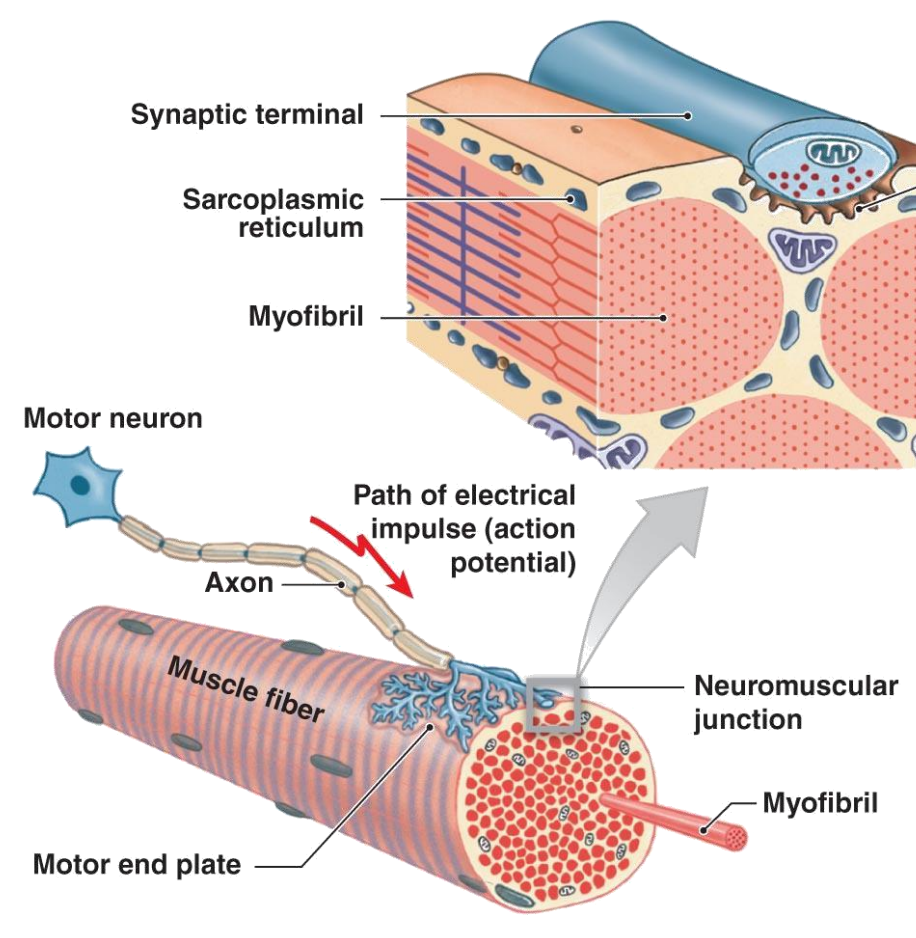

The structural relationship between a skeletal muscle fiber and its lone neuromuscular junction ๑2011 Pearson Education, inc.

Figure $1 \& 2$. Representation of an axon and the neuronal processes involved in a single gesture. 
The inhibition of stimulus diffusion to other parts of the body is the subsequent process of long maturation in the evolutionary path. The first intentional motor acts tend to manifest themselves with a global somatic participation, due to the presence of involuntary overtones (syncinesies) more or less widespread to body parts not involved in the motor command. The syncinesies can be of a tonic or imitative type, widespread or limited to some parts, axial, proximal or distal.

Somatic integration is the maturing process that harmonizes and makes intentional action optimal. The limitation of diffusion is fundamental for the organization of complex motor schemes that engage somatic parts not directly involved in the execution of the programmed act, these somatic parts perform aid and reinforcement functions (utility synergisms) to the intentional motor action. A typical example is the adaptation of the trunk, the lower limbs and the upper limb contralateral to the limb which strongly launches an object. These synergisms require spatial organizations and executive times integrated with each other in a somatic harmony aimed at obtaining the best possible result. The grafting of the various synergisms takes place with progression in the evolutionary course and depends on the motivation, the quality and quantity of experiences. The appearance of synergies is usually automatic, unless there is an instruction like in sports.

In addition to this type of somatic integration, which we could call aid and reinforcement integration to a significant segmentary act for intentional action, we must take into account those integration processes between two or more body parts that perform actions among themselves. differentiated, but rightly combined with each other to create an action, for example screwing and unscrewing with both hands, knitting, combined use of a knife and fork, etc ...; this could be called "collaboration integration".

Rosano and Galletti (1980) owe the attention to the attention and the deepening of the research on the organization of the motor action. The authors examining the evolutionary variability of the motor action patterns, have found a recurrence of certain dysfunctions and have confirmed the already known paratonic disorders (typical of motor weakness), attention disorders (typical of psychomotor instability) and have proposed the recognition of a new dysfunction: disturbances in the process of integration of the body segments for a functional whole of the act. To this type of dysfunction, they have proposed the term clumsiness and the possibility of recognizing the existence of one nosographic entity well defined and frameable in the maldestrezza syndrome.

The syndromic diagnosis is based on a typical motor organization with the exclusion of any other motor disorder; the basic motor schemes are free and the simple motor acts, not requiring a process of somatic integration, are adequate for the purpose. The pathogenic nucleus consists of the deficiency of integrative adaptation of the whole soma or of some parts to the motor activity intentionally performed by a body segment or the lack of integration between the two hemisomes or between the upper and lower limbs or between the limbs. and the trunk or be a global deficit. The most probable cause of the deficit can be hypothesized in a difficulty of evolutionary progression of inhibitory processes, such as to frustrate or limit the process of somatic integration.

Previously the term "clumsiness" has entered our linguistic heritage to signify an inadequate use of the dominant hand or a generic inadequacy in the correct execution of the movement; it is often used to indicate an unspecified movement disorder. French authors also use the term maladresse for a general disorder of motor activity supported by different causes. Wallon, in one of his works in 1928, writes that the clinical symptom "clumsiness" can be sustained by five different causes: cerebellar insufficiency, deficiency of the striatum, difficulty in excluding the representative intervention of the act during the execution of the already automated acquisition of the same, a pragmatic-gnosic disorder or an exalted state of emotion typical of early 
evolutionary phases. Today, as a result of greater knowledge, it is no longer possible to accept the confusing and general use of terms, therefore it is desirable that the importance of a correct use of terms be recognized, many of which are due to neurological dysfunction.

In addition to the clumsy syndrome, the clumsy symptom that can be present in several other syndromes is also recognizable.

The symptomatology is different depending on the case.

Symptoms depending on the lack of integration between the two hemisomes (Rosano and Galletti). There is the difficulty, sometimes the impossibility, of integrating the contralateral segments with those that are carrying out an action. The methods of spatial adaptation are organized as if the individual lived two hemispaces each managed by the corresponding body; the most frequent symptoms are: difficulty in jumping on one foot, kicking the ball, going up and down stairs with alternating feet, performing asynchronous movements with the two feet, imitating asymmetrical gestures, alternating dribbling with both hands, to perform any work that requires the simultaneous and differentiated use of the two hands, to reproduce asymmetric drawings, to draw horizontal lines that engage the two hemispaces of a large sheet.

Symptoms dependent on the lack of integration between upper and lower limbs (scapular girdle and pelvic girdle) (Rosano and Galletti) All those global motor activities that require a synergistic and differentiated participation of the four limbs are compromised; the most characteristic symptoms are: shortage and arrhythmia of the pendulum movements of the upper limbs during running and running, poor synergistic adaptation of the upper limbs during the jump and the kick of the ball, scramble running slowly and with arrhythmic movements, difficulty overcoming obstacles that engage the contemporary and differentiated use of the four arts.

This interesting research has stimulated a review of my case studies that allowed us to identify some syndromic pictures related to the clumsy syndrome described by Rosano and Galletti. Deepening my studies (1981-1990) I could observe that in some cases there is also a poor adaptability of the trunk in the changes of posture and in the global movements of the soma, such as to determine a global lack of the somatic integration process. The latter type has a decidedly greater frequency than the previously described forms. For this reason, I have proposed the recognition of a type of global deficit of the process of somatic integration.

Symptoms dependent on the deficit of global integration (Russo, 1986). The most evident deficiencies are found in the race, in the jump, in the postural passages, in the crawling march, in overcoming obstacles, in football and in throwing the ball. The trunk presents adaptive difficulties making precarious the action of the limbs that present, differently expressed, also typical symptoms of the disturbance between the two hemisomes and between the two tracks. In the less compromised cases there may be attempts at somatic integration, but with results that compromise the economy and the harmony of the motor action and sometimes also its validity. In individuals with global clumsiness in most cases there are no specific relational problems in the psychomotor period. In the evolution there is a slow and partial compensation of the deficit, but there is a progressive lack or lack of availability for gymnastic and sporting activities and a basic tendency to expose little in the group of peers in complex motor activities, an attitude imputable to a progressive taking of awareness of one's own deficit. 
In the syndrome the higher psychic functions are not compromised and so is the gross knowledge of one's static and dynamic body model; on the contrary, in the form of deficit of integration of hemisomes there is a deficient organization of orientation of the body in space, a prevalent use of selected motor patterns for certain situations and a scarce possibility of immediate adaptive choice to varying situations that require complex and global activities. motor. Particularly in the deficit between the two hemisomes, these are acted in homologous hemispaces, delaying the lack of synthesis in a single space It is conceivable that this dual spatial and body duality makes the spatial and symbolic processes of synthesis more complex.

The disturbances of integration between the two hemisomes, between the two tracks and between the trunk and the distal segments, can occur individually or variously combined. The integration deficit syndrome is not very frequent and among all the forms of awkwardness that between the two hemisomes is rare. For the latter in three cases I found for the drawing the use of the left hand in the left hemispace of the sheet and the right in the right hemispace of the sheet. The symptom clumsiness is frequently found in various syndromes and is always present in the syndrome of insufficient motor inhibition.

\section{THERAPY}

Respecting the setting and the psychomotor setting, it will have to provide different objectives depending on the integrative deficit, but the principle will always be that of the conquest of the synergistic capacities of the whole body in complex motor activities.

In the integrative deficit between the two hemisomes, in the first months of therapy and taking advantage of relaxation techniques, ample space should be given to the body's awareness in different postures, first symmetrical and subsequently asymmetric. This preliminary work allows the strengthening of the contemporary perception of the two hemisomes and prepares for the attentive availability of the use of a body globality.

Subsequently, symmetrical segmented motor acts, limited to an upper or lower limb, performed on one side only and alternated, with pauses of a few seconds, will be required for the same acts performed with the contralateral segment of the same name. In progression the complexity of the motor action will be increased. In later times the pause will be reduced until it is cancelled in order to create alternate symmetrical movements, first simple and then more complex. Once enough harmony has been achieved in this work, both for the upper and lower limbs, we can begin, with the same progression mentioned above, to propose asymmetrical movements with two homologous arts, first alternating and then simultaneously.

The execution of these activities must be carried out in privileged positions (supine or sitting) that do not fully engage the body and therefore do not require the simultaneous intervention of complex functions (example: the antigravity) to be integrated with intentional acts.

The final phase of the intervention will be the objective of favouring the harmonization of the two hemisomes in global movements of the whole body. The following activities may be considered: walking and running due to the presence and rhythm of the pendular synergic movements of the upper limbs, the hopping on one foot only due to the differentiated implication of the contralateral hemisome, the throwing of the ball with a hand for the intervention of the contralateral upper limb of reinforcement to the anterior projection of the shoulder ipsilateral to the launch, the cross-toed crawl and the cross smear for the alternation of the differentiated use of the four limbs, the overcoming of various obstacles, the rapid passage of postures and every other activity that engages the two hemisomes in global movements of the whole body. 
In the lack of integration between the tracks the progression is similar to the previous one with the difference that the work of differentiation and functional integration will be set between the two limbs and not between the two hemisomes.

The lack of integration between the trunk and the limbs requires a more complex treatment, especially if anomalous postural modalities have been stabilized and containment overtones grafted onto the various activities of global movement. In this deficit the activity of relaxation, control and awareness of one's own axis is indispensable in the different postures and in simple flexion-extension movements, antero-posterior, lateral -lateral and rotation movements. Once we have reached an adequate control and knowledge of our body structure in the different postures, we can begin the request to adapt the trunk to the movements of the limbs, first simple and then progressively more complex.

The treatment of the integration deficit requires the active participation of the patient, therefore, the best results can be obtained in the latency age, but this does not mean that, with appropriate measures and stimuli of desire in game activities, you do not can do a good job even in previous ages.

\section{CONCLUSION}

To cultivate precociously the child to be active constitutes a solid base on which one can structure and "mentalize" one's own character, in such a way that the probabilities of that subject in adulthood of them. Physically and psychologically healthy kings will be higher than any other subject educated in other way; each type of training creates a permanent thing in the individual who is enriched of experiences (Baldacci, 2013) and that's why our proposal derives precisely from the movement, from exercise, from playing the game itself because it is substantial in the evolutionary process of the child, a real and profound effect.

It is therefore essential to take into account what is written, as we have seen, neuromotor conditioning occurs exclusively through a selective search for external stimulations that allow development and learning, "teach the muscle" what to do, where it should do and above all how he must do it.

We must take into account all the moments in which we deny the child the space to play and fun, essential tools for a correct acquisition of motor patterns and for a correct physical independence, which generates in the child security and willingness to take action, consequently in age adult the skeletal muscle system will be calibrated in such a way as to be much more coordinated than a person with awkwardness who has not acquired the correct patterns, and this will greatly make the difference, when you will find yourself in moments of maximum performance research, such as competitions sports or better performance.

\section{REFERENCES}

Bergès, J., Harrison, A., \& Stambak, M. (1965). E'tude de la latéralité: nouvelles perspectives.

Bergès J., Lezine L. (1963). Test of the imitation of gestes, technique of exploration of the body and of the praxies chez the enfant de 3 à 6 ans. Masson, Paris.

Bollea G., Levi G. (1981). The minor neurological dysfunctions in the perspective of H. Wallon. Neurops. Inf. 234, p. 85.

Galifret-Granjon N. (1969). A battery of lateral dominance. In: Zazzo R. «Manual for the psychological examination of the child". Tr. en. (1975) Ed Riuniti, Rome, Vol. 1, pp. 11-40.

Hécaen H. (1959). Dominance, emispherique et préférance manuelle. Evolution Psychiat., 1, 1.

Hécaen H., Ajuriaguerra J. (1956) Le problème de la dominance hémispheriques. J. de Psychol., 473. 
Hécaen H., Ajuriaguerra J. (1963) Les Gauchers. Prevalence manuelle et dominance cérébrale. P.U.F., Paris.

Levi G., Castriota F. (1976). On graphic lateralization. A study of left and right handed children aged 5 to 7 years. Neurops. Inf., 176, p. 109.

Rosano M., Galletti F. (1980). Awkwardness, clumsiness and instability. Rehabilitation notebooks, 12/4, p. 229.

Rosano M., Galletti F. (1981). Specific disorders of psychomotricity in children aged 0-3 years. Early diagnosis, structuring of the body schema, lines of intervention. Proceedings of the I 'Conv. Naz. «Disorders of psychomotricity in age evolutionary. " Salsomaggiore Terme, 26-27, June.

Wallon H. (1928). The maladresse. J. de Psycol. et Path., 1, 61. Reproduced in: Enfance, 1-2.1963. 